\title{
A DERIVATION OF THE GENERALIZED MODEL OF STRAINS DURING BENDING OF METAL TUBES AT BENDING MACHINES
}

\author{
Z. ŚLODERBACH \\ Opole University of Technology \\ Faculty of Applications of Chemistry and Mechanics \\ 45-036 Opole, ul. Luboszycka 7, POLAND \\ E-mail: z.sloderbach@po.opole.pl
}

\begin{abstract}
According to the postulate concerning a local change of the "actual active radius" with a bending angle in the bend zone, a generalized model of strain during metal tube bending was derived. The tubes should be subjected to bending at tube bending machines by the method of wrapping at the rotating template and with the use of a lubricated steel mandrel. The model is represented by three components of strain in the analytic form, including displacement of the neutral axis. Generalization of the model during bending metal tubes at the tube bending machines as compared with the existing papers (Śloderbach, 1999; Śloderbach and Rechul, 2000) consists in including the neutral axis displacement and possibility of determination of strains at each point along the thickness of the wall of the bent tube in the bending and bend zone. The derived scheme of strain satisfies initial and boundary kinematic conditions of the bending process, conditions of continuity and inseparability of strains. The obtained analytic expressions can be classified as acceptable from the kinematic point of view.
\end{abstract}

Key words: bending, metal tubes, bending machine, strains, neutral axis.

\section{Introduction}

Tube bending see e.g. (Beskin, 1945; Boyle, 1971; Franz, 1961; 1969; Gruner, 1960; Grunow, 1985; Korzemski, 1968; 1971; Li et al., 2006; Pesak, 1953; Śloderbach et al., 1999; 2000; 2002; 2008; 2012; Tang, 2000; Wick et al., 2001; Yang and Lin, 2004; Zdankiewicz, 1970; 1998; Zhang et al., 2011; Zhiqiang et al., 2011) as a technological problem appeared in the end of the 19-th century when production of tubes started in an industrial scale. Tubes were delivered mainly to industry of steam engines and boilers, gas engineering, power engineering, civil engineering. At present, tubes and elbows are purchased by almost all branches of industry, and tube bending is a typical activity in many technological processes in metal industry. Production of tubes and elbows has been increased, and now it is increasing more quickly than the production of steel because tubes and elbows are made also of other materials, for example plastics.

We can also observe higher and higher requirements concerning the quality of the produced tubes and elbows, i.e., production accuracy is higher, tolerance of non-uniformity of the wall thickness distribution, corrugations and so on decrease. We can also talk about higher requirements concerning technological activities, such as tube bending, elongating, necking, beveling etc. Selection of a tube bending method is dependent on the kind of material used, thickness of the tube, bending radius, the required accuracy and quality of bending, work conditions, bend angle, serial production and others.

At present, tube bending at tube bending machines using the method of wrapping at the rotating template with the lubricated mandrel is the most widely used. Such bending always leads to the formation of thinner walls in the layers subjected to elongation, thickening and wrinkling (corrugation) in the layers subjected to compression, and deformation (ovalization) of the cross section. Such unfavorable phenomena should be included in the limits of tolerance given in European standards and recommendations (European Standard, 1993; Zdankiewicz, 1998), as well as regulations of UDT [the Polish Office of Technical Inspection, (UDT Conditions, 2003)]. The acceptable ovalization of the cross section according to the European Standard (EN 448, 1993) is up to $6 \%$. In this paper, the authors consider only cold bending of 
metal tubes of the assumed technological wall thickness $s^{*} \leq 0.10$ and maximal $d_{\mathrm{ext}}=160 \mathrm{~mm}$ (Śloderbach, 2012), (where $s^{*}=g_{0} / d_{\mathrm{ext}}, g_{0}$ and $d_{\mathrm{ext}}$ - initial thickness and external diameter of the bent tube, respectively). In regulations of UDT (UDT Conditions, 2003), pressure tubes are assumed as thin-walled, when $s_{w}^{*} \leq 0.05$, where $s_{w}^{*}=g_{0} / d_{\text {int }}, d_{\text {int }}=d_{\text {ext }}-2 g_{0}$, then $s_{w}^{*}=s^{*} /\left(1-2 s^{*}\right)$. The above assumptions follow from the practical results obtained in the Research and Development Institute for Power Plant Maintenance in Wrocław, Poland (it stopped to exist in 2006). Tubes of the external diameter up to $160 \mathrm{~mm}$ and wall thickness $s^{*} \leq 0.1$ were technologically cold bent at the bending machines with the rotating template with the use of a lubricated rigid metal mandrel. Greater metallic tubes (with greater $d_{\mathrm{ext}}$ and $s^{*}$ ) are bent by hot, half hot or with preheating methods (Śloderbach, 2002).

The main reasons influencing a shorter service life of elbows are: decrease of the wall thickness in the elongated layers, complex and unfavourable states of external loadings and internal stresses, large plastic strains forming while tube bending, storage of energy of plastic deformation and its influence on internal structure of the material, and creep resistance. Damages of elbows occurring during service of pipelines and tube installations can also be caused by unsufficient reliability of the applied method of strength calculations (Śloderbach and Rechul, 2000; Śloderbach, 2002; Tang, 2000; Zhiqiang et al., 2011). Strictly speaking, there were no accurate methods of determination of permissible distribution of wall thickness in the points of maximum strains in the elbow bending zone. It concerns especially the tip parts of the bending zone where the thickness is minimum. If we know components and intensities of strain in the bending zone, especially in the tip part, we will be able to make more accurate strength calculations. In consequence, we will be able to improve reliability of devices containing elbows.

The description presented herein concerns tube bending at the bending machines with the use of the method of wrapping the bent tube on the rotating template with a segment elastic lubricated mandrel (see Fig.1). This method is widely known and used.

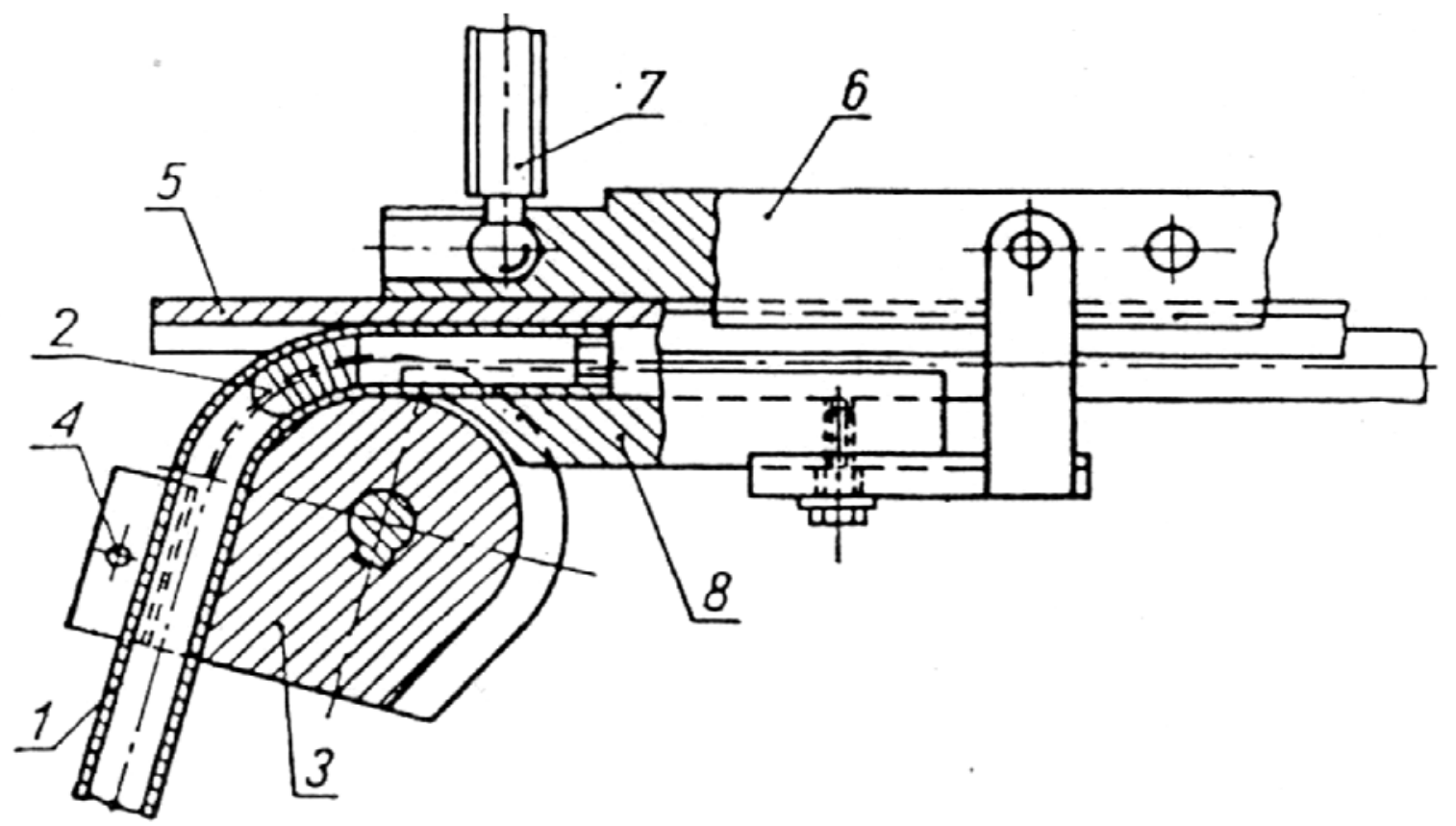

Fig.1. Scheme of a tube bending machine with a rotating template and a flexure mandrel, 1- bent tube, 2flexible segment mandrel, 3- rotating template, 4- clamping jaws, 5- clamping strip, 6- guide, 7screw for regulation of clamping force of the strip and the planisher, 8-planisher. 
A correct analysis of plastic strains in the elbow bending zone, especially in the elongated layers is very important from the point of view of strength, also for service, technological, economic and safety reasons.

The main aim of this paper is to derive basic relationships for component measurements of strains including displacement of the neutral axis during bending metal tubes at bending machines by the wrapping method at the rotating template and using a template and a mandrel (or without a mandrel). Almost a circular shape of the internal surface of the bent elbow (no Brazier effect), and the assumption that $d_{\text {int }}=$ const must be kept. At present, this method is widely used for the production of most metal elbows (Beskin, 1945; Boyle, 1971; Franz, 1961; Gruner, 1960; Grunow, 1985; Korzemski, 1968; 1971; Śloderbach et al., 1999; 2002; Wick et al., 2001; Zdankiewicz, 1998) for many tube installations. This method concerns especially bending of the tubes at the radii $\left(R>1.5 \times d_{\text {ext }}\right)$ being thin-walled $\left(s_{w}^{*} \leq 0.05\right)$ according to (UDT Conditions, 2003) and thick-walled $s_{w}^{*}>0.05$. Metallic elbows manufactured in such a way are usually applied as elemnts of pipelines in devices for power engineering and some other fields of industry. Tube bending at the radii $(R$ $\left.<1.5 \times d_{\text {ext }}\right)$ is performed by other methods, and tubes of very thin walls $\left(s_{w}^{*}<<0.05\right)$ are usually not applied in industrial pressure installations.

In literature there are no analytic expressions for strains depending on an actual value of the bending angle, angles determining a position of each point (particle) in the bending and bend zone, displacement of the neutral axis, the bending radius, dimensions of the cross section of the bent tube and suitable technological-material coefficients $\left(k\right.$, and $\left.\lambda_{i}\right)$ dependent on bending technological parameters and tube materials. Tube bending always leads to a reduction of the wall in elongated layers and an increase of tube thickness in layers subjected to compression, to ovalization and formation of corrugation which distort the cross section. The ovalization effect occurs as a consequence of oblateness of the cross section (Brazier effect), non-uniform reduction and thickening of walls of the bent elbow, and the corrugation effect. Such unfavourable phenomena, i.e., changes of the tube wall thickness and wrinkling, and also ovalization of the cross section shape should be included in the limits of permissible tolerances (European Standard, 1993; UDT Conditions, 2003; Zdankiewicz, 1998).

In this paper, the author tried to describe the strain state understanding tube bending as a threedimensional, heterogeneous (including changes of the strain state with the bending angle and the angles of the point position in the bending and bend zones) and including the influence of the neutral axis displacement problem. Such influence is understood in the following way: the forming field of plastic strains generates a displacement of the neutral axis (in this bending method it is a downward displacement in the direction of the layers subjected to compression), and the quantity of the neutral axis displacement affects the distribution of these strains.

From experiments, technological tests and industrial practice (Wick et al., 2001) it appears that this displacement is only about $5 \%$ value of the external diameter of the bent tube for thick-walled tubes $\left(s^{*} \geq\right.$ $0.10)$ bent at relatively large bending radii $\left(R>2 \times d_{\text {ext }}\right)$, and about $25 \%$ for very thin-walled tubes $\left(s^{*} \leq 0.01\right)$ bent at very small bending radii $\left(R \leq 1 \times d_{\text {ext }}\right)$. Thus, such a high displacement of the neutral axis of plastic bending should not be disregarded in an analytic description of the strain state. Generalization of the strain description while bending metal tubes at bending machines related to the data from other papers (Śloderbach, 1999 ; 2000) consists in including the neutral axis displacement and possibility of determination of strains in each point of the tube wall in the bending and bend zones.

In the derivation of the model for the field of strain all the thermal effects accompanying large deformations were disregarded. It is assumed that the tube bending process at bending machines is quasiisothermal. The model of tube bending was obtained on the assumption that the strain process in the layers subjected to tension and compression is quasi-static, so dynamic effects were omitted (Śloderbach et al., 2006; 2008).

It is assumed that strains in the tube bending process are identified with plastic strains. Thus, it appears that plastic strains are of the order of some tens of percent (even to 50\%), and the maximum elastic strains are equal to decimal parts of the percent and they are neglected in an analytic description. In the angular measure of the bending process, only some degrees concern elastic strains. 


\section{Geometric-analytic description of the bending process}
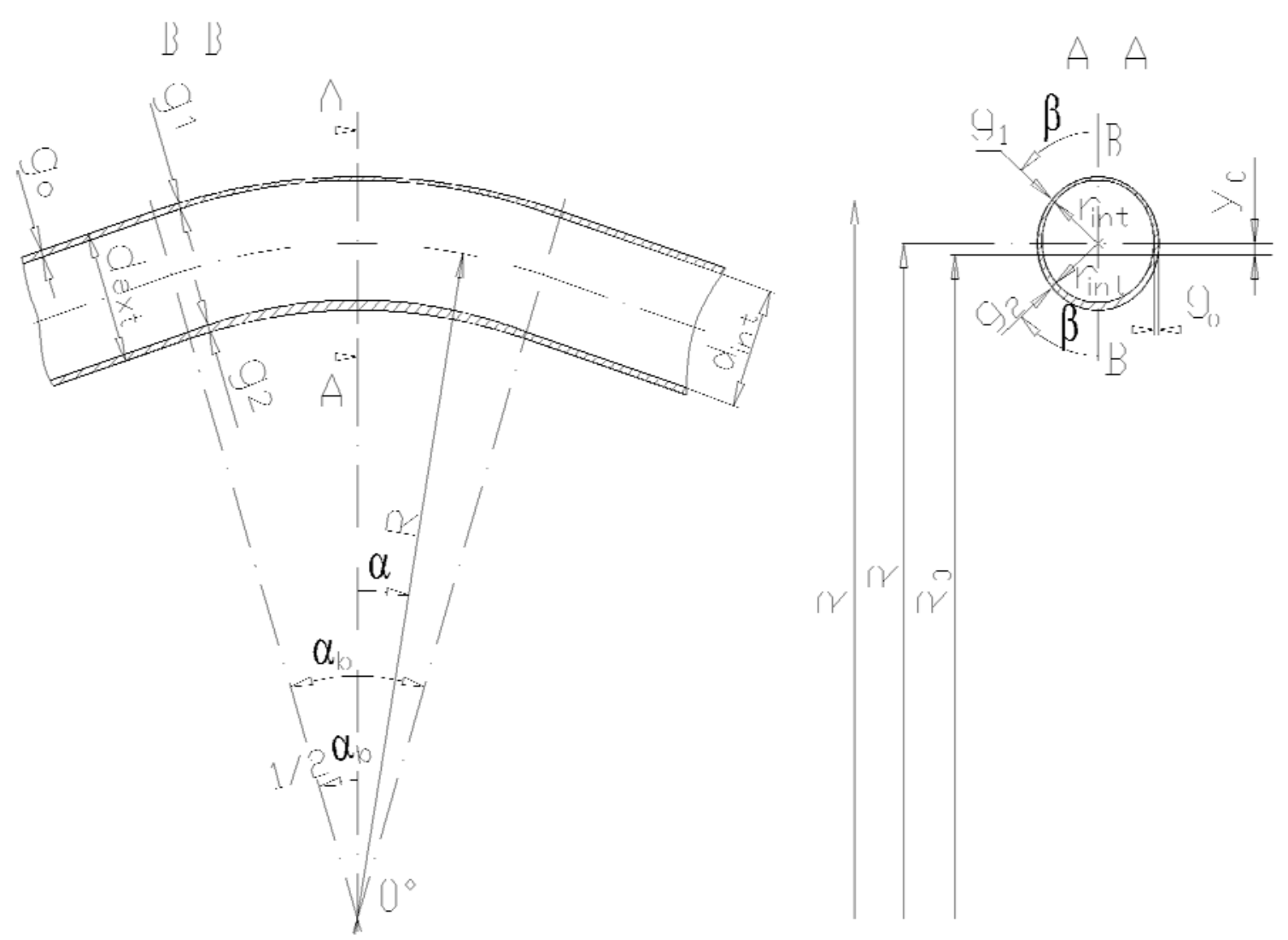

Fig.2. Geometrical and dimensional quantities pertaining to tube-bending processes.

\section{The basic assumptions}

It is assumed that the tube material is an incompressible rigid-plastic (with isothropic hardening) continuous medium satisfying the condition of plasticity $(\mathrm{M}-\mathrm{H}-\mathrm{H})$ and the Levy-Mises flow laws. Its properties while bending are described by two technological-material coefficients $\left(k\right.$ and $\left.\lambda_{i}\right)$. Thus, the constant plastic volume of the material is assumed before and after bending. The neutral axis of plastic bending is determined by the radius $R_{0}$. The axis separating elongated and compressed layers in the bending zone is a line of stress discontinuity (Hill, 1986; Johnson and Mellor, 1975; Marciniak, 1971; Mendelson, 1988; Olszak et al., 1985; Szczepinski, 1973; Śloderbach, 1999, 2002; Tang, 2000). The strains on the axis with the radius $R_{0}$ and at the beginning and the end of the bending zone are equal to zero. In the paper it is also assumed that in the tip points of the elongated layers (environment of the mandrel), the tube bending process at the bending machines (tube bending by wrapping at the rotational template and with the use of a lubricated mandrel) is a complex process of heterogeneous curvilinear elongation (biaxial stretch drawing) under the three dimensional stress state (spatial while bending thick-walled tubes, for example when $s_{w}^{*}>$ 0.05 , and plane while bending thin-walled tubes). In the case of the compressed layers, it is a composition of two processes: the heterogeneous curvilinear compression and unfree upsetting. Moreover, it is assumed that the internal section of the tube remains circular, so a deformation in the form of the cross section flattening 
is not taken into account. It can be a result of the influence of a suitable and well manufactured mandrel (for example a flexural and segment one) and its precise set-up while tube bending. As introduction of the technological-material coefficient $k$ allows to include (in part and indirectly) also the influence of mandrel friction to the tube wall on strain distribution.

In real tube bending processes at bending machines, in the compressed layers there are no such strain states as those occurring under free compression and upsetting. Tube bending is not a case of free (unbounded) bending but it is forced by the structure of working tools of the bending machine (matrix, mandrel, flatter, strip and its pressure force). There are some boundary limitations of displacements resulting from the bending machine structure, forces of external friction of the material and the bending machine tools, clearances between the walls of the bent tube and the tools. The amount and quality of lubrication is of a great importance, too.

\section{Procedure of strain component derivation}

The generalized expressions for three components of the strain state for the considered tube bending at bending machines are going to be derived at three stages:

1. Derivation of the expressions for principal strains in relative and next in logarithmic measures while bending when the influence of the neutral axis displacement on the strain distribution is not taken into account,

2. Derivation of the expression for the neutral axis displacement of plastic bending $y_{0}$,

3. Formal derivation (according to Fig.4) of expressions for strain components in order to describe the deformation state for the problem including the neutral axis displacement by an introduction of the derived relationship for $y_{0}$ into the equations.

\subsection{Derivation of expression with no neutral axis displacement}

Ad.1. The basic aim is an analytic derivation of the plastic strain components, i.e., the longitudinal (along the tube axis), circumferential and radial (along the thickness) components in measures of relative and logarithmic strains during tube bending at bending machines by wrapping at the rotating template using the mandrel or with no mandrel, on the assumption that $d_{\text {int }} \cong$ const. It concerns thin-walled and thick-walled metallic tubes subjected to cold bending. The expressions determining the strain components have been derived on the assumption of plane sections (simple bending) for an incompressible plastic material. This method allows determination of the strain state at each material point included in the bending and bend zone. Such an analytic strain scheme satisfies kinematic conditions of the process, i.e., initial and boundary conditions of the tube bending process, conditions of continuity of strains and displacement conformity, and incompressibility of the material, like in the method of selection of kinematically permissible fields of strains or velocity fields of strain (Hill, 1986; Olszak et al., 1985; Szczepinski, 1973; Śloderbach, 1999; 2002; Życzkowski, 1981).

An important problem is to determine a form for a change of "actual active radius" $r^{*(N)}$ in the bending zone (determined at each $N$-th point along the thickness, and for total thickness of the bent tube, so as $\left.r^{*}=\left.r^{*(N)}\right|_{g_{0}^{(N)}=g_{0}}\right)$, depending on a change of the bending angle $\alpha_{b}$. The increase related to length of the bending radius $R$, defines an increment of the relative longitudinal (along the tube axis) strain $d \varepsilon_{1}^{(N)}$. The local "actual active radii $\left(r^{*(N)}\right.$ and $\left.r^{*}\right)$ depend on the actual value of the angles $\alpha$ and $\beta$ determining positions of the points in the bending zone, and on the actual position of the external point in the considered $N$-th layer included in the wall of the bent tube (see Fig.3), also on total dimensions of the cross section in the elongated and compressed layers. 

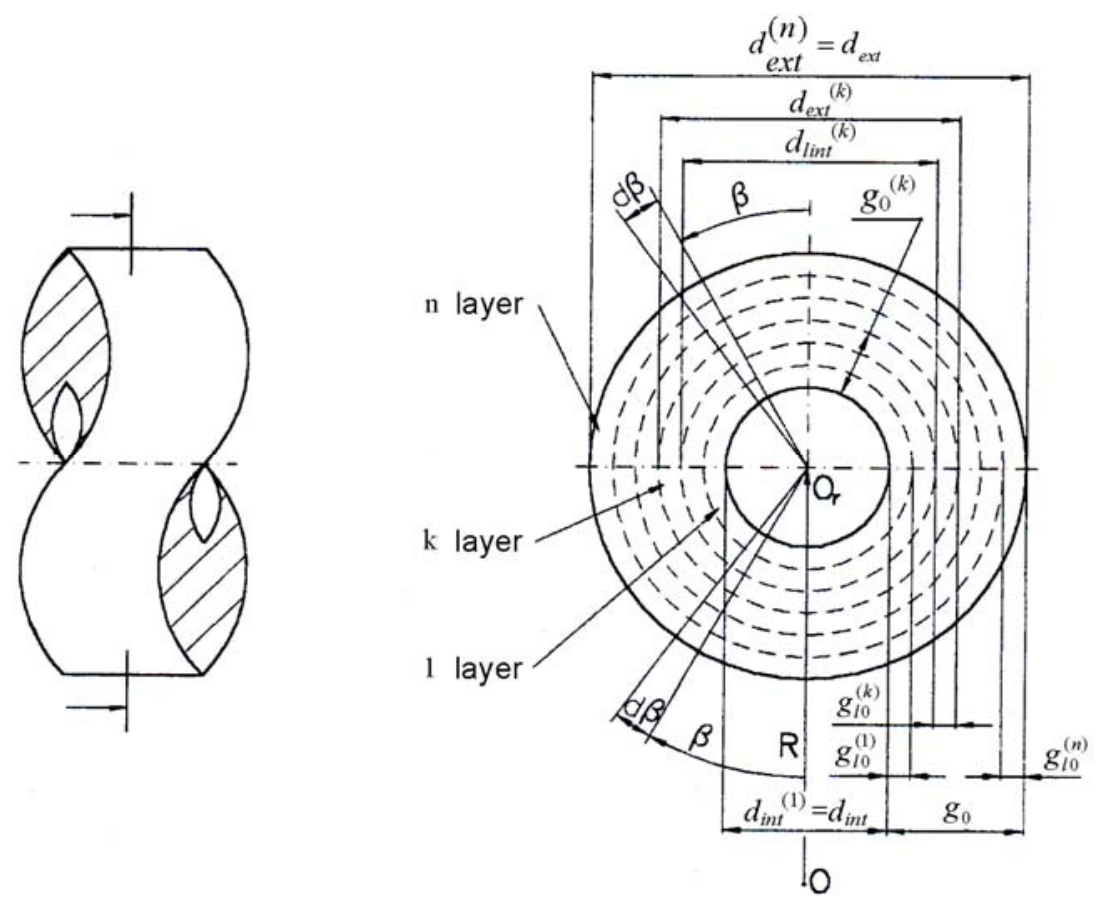

Fig.3. A concept of division of the transversal section of a thick walled pipe for the analytical and FEM method.

In the analytic method, a possibile number of the analyzed points along the thickness of the bent tube wall is $N \in\langle 1 ; n)$, when $n \rightarrow \infty$. In this method, a division along the wall thickness is laminar into the $N$-th number of layers $g_{l 0}^{(N)}$ in thickness, thickness of which is measured from the internal tube surface to the external point of the $N$-th layer (see Figs 2 and 3), where $g_{0}^{(k)}=\frac{d_{\mathrm{ext}}^{(k)}-d_{\mathrm{int}}}{2}=r_{\mathrm{ext}}^{(k)}-r_{\mathrm{int}}$. Such division results from the fact that tube dimensions are given as $\left(l \times d_{\mathrm{ext}} \times g_{0}\right)$, where $l-$ tube length. When $N=\langle 1\rangle$, then $g_{l 0}^{(I)}=g_{0}$, and when $N \rightarrow \infty$, then $g_{l 0}^{(N)} \rightarrow 0$. In the case of the finite element method (FEM) applied for the thick walled tubes, the division is annular into a finite number of cylinders (rings) $n$ of the equal thickness. From Fig. 3 it also appears that when the wall thickness is considered as one layer for $N=\langle 1\rangle$, then $g_{l 0}^{(l)}=g_{0}^{(l)}=g_{0}, d_{\text {int }}^{(l)}=d_{\text {int }}$ and $d_{\text {ext }}^{(l)}=d_{\text {ext }}$, where $d_{\text {ext }}=d_{\text {int }}+2 g_{0}$.

The value of the modified relative strain $\varepsilon_{l}$, (i.e., the strain corresponding to the experimental results) obtained after previous integration of the expression for $d \varepsilon_{l}$ within the limits of angle $\left\langle 2 \alpha ; \alpha_{b}\right\rangle$ depends on the actual value of the bending angle $\alpha_{b}$, actual values of the point position angles $\alpha, \beta$ in the bending zone, actual dimensions of the bent tube in the elongated and compressed layers, the bending radius $R$, and suitable correction technological-material coefficients $k$ and $\lambda_{i}$. Let us describe the strain state assuming the following postulate:

\section{$\underline{\text { Postulate } \mathbf{A}^{*}}$}

An increment of the longitudinal component (along the tube axis) $d \varepsilon_{1}^{(N)}$ of the strain state, according to the notations in Figs 2 and 3 (tube bending at bending machines by using the mandrel, or with no mandrel but keeping the constant internal diameter $d_{\text {int }} \approx$ const), for the external point of every $N$-th layer included in 
the tube wall is directly connected with an increment of "the local active actual radius" $r^{*(N)}$, and increments of circumferential and radial strains $d \varepsilon_{2}^{(N)}$ and $d \varepsilon_{3}^{(N)}$ are presented in the following way

$$
\begin{aligned}
& d \boldsymbol{\varepsilon}_{l}^{(N)}= \pm \frac{d \boldsymbol{r}^{*(N)}}{|\boldsymbol{R}|}, \quad d \boldsymbol{\varepsilon}_{l}=\left.d \boldsymbol{\varepsilon}_{1}^{(N)}\right|_{g_{0}^{(N)}=g_{0}} \quad \text { and } \\
& d \boldsymbol{\varepsilon}_{2}^{(N)}=\frac{d \boldsymbol{r}_{i}^{(N)}}{\left|\boldsymbol{r}_{\text {ext }}^{(N)}\right|} \quad \text { and } \quad d \boldsymbol{\varepsilon}_{3}^{(N)}=\frac{d \boldsymbol{g}_{i}^{(N)}}{\left|\boldsymbol{g}_{0}^{(N)}\right|}
\end{aligned}
$$

where $d \boldsymbol{r}^{*}=\left.\left[d \boldsymbol{r}^{*(N)}=\left(\boldsymbol{s}_{\alpha b}^{(N)} \times \frac{d \boldsymbol{\alpha}_{b}}{2}\right) \cos \beta\right]\right|_{g_{0}^{(N)}=g_{0}}$, because when $N=\langle 1\rangle$, then $g_{0}^{(N)}=g_{0}$ and the sign $(+)$ is related to the elongated layers, and (-) to the compressed ones.

For the division of a thick-walled tube into a finite number of cylinders (rings) prepared for the FEM method, we take

$$
d \boldsymbol{\varepsilon}_{l}^{(N)}= \pm \frac{d \boldsymbol{r}^{*(N)}}{|\boldsymbol{R}|}, \quad d \boldsymbol{\varepsilon}_{2}^{(N)}=\frac{d \boldsymbol{r}_{i}^{(N)}}{\left|\boldsymbol{r}_{\text {ext }}^{(N)}\right|}, \quad d \boldsymbol{\varepsilon}_{3}^{(N)}=\frac{d \boldsymbol{g}_{l i}^{(N)}}{\left|\boldsymbol{g}_{l 0}^{(N)}\right|} \quad \text { and } \quad g_{i}=\sum_{N=1}^{N=n} g_{l i}^{(N)}
$$

where $g_{i}$ - running thickness of a bend within the bending.

From properties of the vector product it results that

$$
\begin{aligned}
& d \boldsymbol{\varepsilon}_{l}= \pm\left[\frac{\left(\boldsymbol{s}_{\alpha g}^{(N)} \times d \boldsymbol{\alpha}_{g}\right) \cos \beta}{2|\boldsymbol{R}|}=\right. \\
& \left.=\frac{\left(\boldsymbol{r}_{i}^{(N)} \sin \left(\frac{\alpha_{g}}{2}\right) \times d \boldsymbol{\alpha}_{g}\right) \cos \beta}{2|\boldsymbol{R}|}=\frac{\boldsymbol{r}_{i}^{(N)} \cos \beta\left(\sin \left(\frac{\alpha_{g}}{2}\right) \times d \boldsymbol{\alpha}_{g}\right)}{2|\boldsymbol{R}|}\right] g_{0}^{(N)}=g_{0}
\end{aligned}
$$

where $|\boldsymbol{R}| \equiv R$ - length (modulus) of the bending radius and $R=$ const,

and

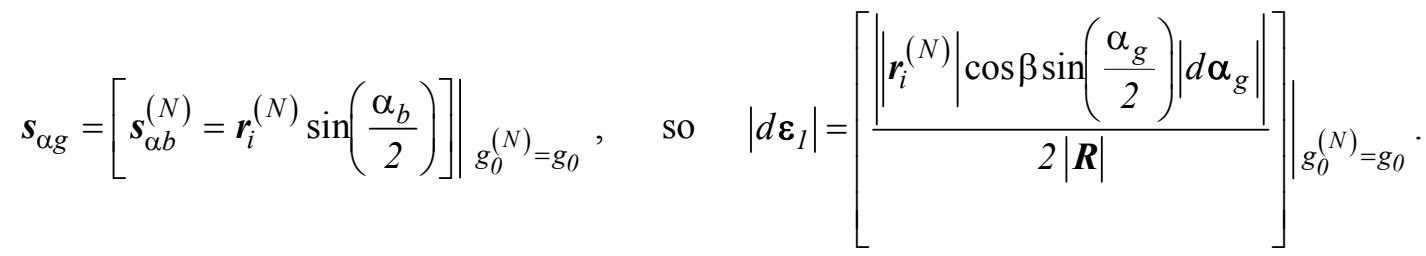

Thus,

$$
d \varepsilon_{1}= \pm\left[\frac{r_{i}^{(N)} \cos \beta \sin \left(\frac{\alpha_{b}}{2}\right) d \alpha_{b}}{2 R}\right] g_{g_{0}^{(N)}=g_{0}}, d \varepsilon_{2}=\left.\frac{d r_{i}^{(N)}}{r_{\mathrm{ext}}^{(N)}}\right|_{g_{0}^{(N)}=g_{0}} \text { and } d \varepsilon_{3}=\left.\frac{d g_{i}^{(N)}}{g_{0}^{(N)}}\right|_{g_{0}^{(N)}=g_{0}}
$$


where $r_{i}^{(N)}$ - local actual radius related to the external point of the $(N$-th) layer included in the tube wall is $r_{i}^{(N)}=r_{\text {int }}+g_{i}^{(N)}$ and $r_{i}^{(N)} \in\left\langle r_{\text {int }} ; r_{i}\right\rangle$.

When: $N=\langle I\rangle$ and when the averaged strains are related to the central layer, then $r_{i}^{(N)} \equiv r_{i m}=r_{\text {int }}+\frac{g_{i}}{2}$, where $r_{i m}$ - averaged small active bending radius related to a half of the actual thickness of the bent tube wall, $r_{\mathrm{ext}}^{(N)}$ - local external radius related to the external point of the (N-th) layer included in the tube wall, $r_{\mathrm{ext}}^{(N)} \in\left(r_{\mathrm{int}} ; r_{\mathrm{ext}}\right)$ and $r_{\mathrm{ext}}^{(N)}=r_{\text {int }}+g_{0}^{(N)}$.

When $N=\langle 1\rangle$, then $r_{\mathrm{ext}}^{(N)}=r_{\text {ext }}$, because $g_{0}^{(N)}=g_{0}$ and $g_{i}^{(N)}=g_{i}$, where $g_{i}$ - local actual thickness of the wall of all the section, $g_{i} \in\left\langle 0 ; g_{0}\right\rangle, g_{0}^{(N)}$ and $g_{i}^{(N)}$ - initial thickness of the considered ( $N$-th) layer of the bent tube wall, such that $g_{0}^{(N)} \in\left\langle 0 ; g_{0}\right\rangle$, and the actual local wall thickness for the external point of the ( $N$-th) layer of the tube section such that $g_{i}^{(N)} \in\left\langle 0 ; g_{i}\right\rangle$, respectively.

A formal notation of expression (4.3) $)_{l}$ in the integral form in the range of a change of angle $\xi$ from the actual value of angle $2 \alpha$ to the value of angle $\alpha_{b}$, i.e., in the range $\xi \in\left\langle 2 \alpha ; \alpha_{b}\right\rangle$, is as follows

$$
\varepsilon_{l}= \pm\left[\int_{2 \alpha}^{\alpha_{b}} \frac{r_{i}^{(N)} \cos \beta \sin \left(\frac{\xi}{2}\right) d \xi}{2 R}=\frac{\cos \beta}{2 R} \int_{2 \alpha}^{\alpha_{b}} r_{i}^{(N)} \sin \left(\frac{\xi}{2}\right) d \xi\right] g_{0}^{(N)}=g_{0}
$$

After formal integration through the parts of $\varepsilon_{l}$, we obtain

$$
\varepsilon_{l}= \pm\left\{\left.\left[\frac{r_{i}^{(N)} \cos \beta\left(\cos \alpha-\cos \left(\frac{\alpha_{b}}{2}\right)\right)}{R}\right]\right|_{g_{0}^{(N)}=g_{0}}+\frac{\cos \beta}{R}\left[\int_{2 \alpha}^{\alpha_{b}} \cos \left(\frac{\xi}{2}\right) \frac{d r_{i}^{(N)}}{d \xi} d \xi\right] \mid g_{0}^{(N)}=g_{0}\right\} \text {. }
$$

As it results from expression Eq.(2.4), the longitudinal component of the plastic strain $\varepsilon_{l}$ (expressed in measures of relative strains) contains two terms. The first term $\pm\left[\frac{r_{i}^{(N)} \cos \beta\left(\cos \alpha-\cos \left(\frac{\alpha_{b}}{2}\right)\right)}{R}\right] g_{g_{0}^{(N)}=g_{0}}$ represents a part of relative longitudinal (along the tube axis) strains $\varepsilon_{l}$, connected with fiber elongation in the elongated layers, and shortening in the compressed layers, respectively depending on the tube dimensions, coordinates of the particle position and the bending radius, as well as on the actual value of the bending angle $\alpha_{b}$.

The other term, $\pm \frac{\cos \beta}{R}\left[\int_{2 \alpha}^{\alpha_{b}} \cos \left(\frac{\xi}{2}\right) \frac{d r_{i}^{(N)}}{d \xi} d \xi\right] g_{0}^{(N)=g_{0}}$ - represents the part of the longitudinal strain (along the axis) $\varepsilon_{l}$ connected with a change of the tube wall thickness, i.e., a reduction of thickness in the elongated layers, and increase of thickness in the compressed layers, respectively. This term value estimated from calculations is negligibly low because its maximum value equals only some per cent as compared with 
the value of the first term. Thus, this term is disregarded in further considerations and calculations, and in consequence the applied procedure and calculations should be simpler. Thus

$$
\varepsilon_{l} \cong \pm\left[\frac{r_{i}^{(N)} \cos \beta\left(\cos \alpha-\cos \left(\frac{\alpha_{b}}{2}\right)\right)}{R}\right] g_{g_{0}^{(N)}=g_{0}} .
$$

The bent tube is a spatial element, so the local description of the stress state should be based on three main components of the plastic deformation state (components: longitudinal (along the axis), circumferential and radial (along the thickness)) obtained after formal integration of expressions Eq.(4.3) in the range of angle $\left\langle 2 \alpha ; \alpha_{b}\right\rangle$. The expression determining the circumferential component was obtained on the assumption that during tube bending material particles are moving along the elbow radius to the center of the bent tube in the elongated layers and from the center in the compressed layers. The actual and averaged local components of the strain state take the following form

$$
\begin{aligned}
& \left.\varepsilon_{1} \cong\left[\varepsilon_{1}^{(N)}= \pm \frac{d_{i}^{(N)} \cos \beta\left(\cos \alpha-\cos \left(\frac{\alpha_{b}}{2}\right)\right)}{2 R}\right]\right|_{g_{0}^{(N)}=g_{0}}, \\
& \varepsilon_{2} \cong\left[\varepsilon_{2}^{(N)}=\frac{d_{i}^{(N)}-d_{\text {ext }}^{(N)}}{d_{\text {ext }}^{(N)}}\right] g_{g_{0}^{(N)}=g_{0}}, \quad \varepsilon_{3} \cong\left[\varepsilon_{3}^{(N)}=\frac{g_{i}^{(N)}-g_{0}^{(N)}}{g_{0}^{(N)}}\right] g_{g_{0}^{(N)}=g_{0}} \text { where: }
\end{aligned}
$$

$d_{i}^{(N)}$ - local actual diameter related to the external point of the $N$-th layer of the bent elbow included in its thickness in the bending zone, $d_{i}^{(N)}=2 r_{i}^{(N)}$,

$d_{\mathrm{ext}}^{(N)}$ - local initial external diameter related to the external point of the $N$-th layer included in the bent tube wall, so as $d_{\mathrm{ext}}^{(N)} \in\left(d_{\mathrm{int}} ; d_{\mathrm{ext}}\right\rangle$ and $d_{\mathrm{ext}}^{(N)}=2 r_{\mathrm{ext}}^{(N)}$ and $r_{\mathrm{ext}}^{(N)}=r_{\mathrm{int}}^{(N)}+g_{0}^{(N)}$ or $r_{\mathrm{ext}}^{(N)}=r_{\mathrm{int}}+g_{0}^{(N)}$,

$g_{0}^{(N)}$ - local initial thickness of the $N$-th layer of the tube included in the bent tube wall so as $g_{0}^{(N)} \in\left(0 ; g_{0}\right\rangle$ and $r_{i}^{(N)}=r_{\text {int }}+g_{i}^{(N)}$,

$g_{i}^{(N)}$ - local actual thickness of the tube layer determined for the $N$-th layer included into the bent elbow wall calculated in measures of relative strains.

Expressions Eq.(2.6) are expressed in local and actual (along thickness) geometrical parameters of the bent tube $\left(d_{\text {int }}^{(N)}, d_{\mathrm{ext}}^{(N)}, g_{i}^{(N)}\right.$ and $\left.g_{0}^{(N)}\right)$. Thus, they can be used for analytical calculations of strain distribution along the tube wall thickness in elongated and compressed layers. In future, the calculation method should be improved and numerical methods using the finite element method should be applied.

The strains should be measured during experiments performed in order to verify analytical or numerical calculations. Thus, transformation of Eqs (4.6) for the determination of strains for the total thickness of the elbow wall (it concerns especially thin-walled tubes) measured on external surfaces (external measuring quantities after bending), or measurements of the initial thickness of the tube which is going to be bent requires averaging and replacement of the values $d_{\mathrm{ext}}^{(N)}$ from Eq.(4.6) by $d_{\mathrm{ext}}$, and $g_{0}^{(N)} g_{0}$, because when $N=\langle 1\rangle$ then $g_{0}^{(N)}=g_{0}, d_{\mathrm{ext}}^{(N)}=d_{\mathrm{ext}}, d_{i}^{(N)}=d_{i}$. Now, it is necessary to derive measures of the logarithmic 
strains, useful in plastic work technologies for the case of large strains (Franz, 1961; Hill, 1986; Johnson and Mellor, 1975; Marciniak, 1971; Mendelson, 1988; Śloderbach, 1999; 2002; Życzkowski, 1981), as

$$
\begin{aligned}
& \varphi_{1}=\ln \frac{2 R \pm d_{i} \cos \beta\left(\cos (\alpha)-\cos \left(\frac{\alpha_{b}}{2}\right)\right)}{2 R}, \\
& \varphi_{2}=\ln \frac{d_{i}}{d_{\text {ext }}}=\ln \frac{d_{\text {int }}+2 g_{i}}{d_{\text {ext }}}, \quad \varphi_{3}=\ln \frac{g_{i}}{g_{0}} .
\end{aligned}
$$

The expressions for component strains Eqs (4.1) - (4.7) describe deformation in bending tubes made of plastic and incompressible continuous media (Śloderbach, 1999; 2002). Real materials undergo a deformation in another way (especially in the compressed layers), and tube bending at bending machines is not unbounded upsetting in those layers. There are boundary limitations (especially in the compressed layers of the bent tube) resulting from the bending machine structure and its rigidity.

There are also forces of external friction of the tube with the bending machine tools, and internal friction in the bent tube materials, as well as elastic strains of the bending machines from bending moments, tensile and compressive forces, and others. From tests described in literature (Franz, 1961; Gruner, 1960; Grunow, 1985; Korzemski, 1968; 1971; Wick et al., 2001; Yang and Lin, 2004; Zhang et al., 2011; Zhiqiang et al., 2011) and the tests performed by the author (Sloderbach, 1999; 2000; 2002) it appears that expressions Eqs (4.1)-(4.7) should be modified. Only the longitudinal component (along the tube axis) should be modified because values of the circumferential and radial components (along the thickness) result directly from a value of the longitudinal component and the condition of plastic incompressibility of the material. The longitudinal component expressed in logarithmic (real) measures of strains Eq.(4.7) was modified because the results of experimental measurements are defined in such measures and determined on the external surfaces of the bent tube (Franz, 1961; Gruner, 1960; Grunow, 1985; Korzemski, 1968; 1971; Śloderbach, 1999; 2000; 2002).

Modification of expressions Eq.(4.7) consists in an introduction - according to the experimental data - of two parameters $k$ and $\lambda_{i}$ (technological-material parameters of the tube bending process) for the case $N=$ $<1>$. Thus

$$
\begin{aligned}
& \varphi_{1}=\lambda_{i} \ln \frac{2 R \pm d_{i} \cos \beta\left(\cos (k \alpha)-\cos \left(k \frac{\alpha_{b}}{2}\right)\right)}{2 R}, \\
& \varphi_{2}=\ln \frac{d_{i}}{d_{\mathrm{ext}}}=\ln \frac{d_{i n}+2 g_{i}}{d_{\mathrm{ext}}}, \quad \varphi_{3}=\ln \frac{g_{i}}{g_{0}}
\end{aligned}
$$

where $g_{i}$ - local actual thickness of the bent elbow wall,

$d_{i}$ - local actual external diameter of the elbow in the bending zone: $d_{i}=2 r_{i}, d_{i}=d_{i n t}+2 g_{i}$,

$\lambda_{i}$ - technological-material coefficient of strain distribution in the elongated layers $(i=1)$ and compressed layers $(i=2)$, defined from experiments, so as $\left(\lambda_{1} \cong 1\right.$ and $\left.\lambda_{2} \in\langle 0,1\rangle\right)$. In most cases of known tests on the tube bending process using the method considered in this paper it can be assumed that $\lambda_{2} \approx 0.5$,

$k$ - technological-material coefficient dependent on the bent tube material and the applied bending technology, determining a bending zone range in the bent zone. This coefficient is defined during experiments, theoretically $k \in\langle 1 ; \infty)$. It seems that in the case of most of metallic materials it is sufficient when $k \in\langle 1 ; 6\rangle$. 
From the tests and calculations it even appears that $k \in\langle 1 ; 3\rangle$, see e.g. (Franz, 1961; Gruner, 1960; Grunow, 1985; Korzemski, 1968; 1971; Śloderbach, 1999; 2000; 2002). In the case of more ductile, soft, plastic materials bent at elevated temperatures (hot, semi-hot or preheated bending) bent with a greater radius $R$ and at a more fitted expanding mandrel (segment, with an adjusted external diameter) with rich lubrication of the mandrel and the tube interior, the coefficient $k$ is lower (tends to the unit, $k \rightarrow 1$ ).

Thus, it appears that the coefficient $k$ allows including (indirectly and in part) some effects of friction between the mandrel and the bent tube wall. For elbows bent to $180^{\circ}$, the coefficient $k$ expresses a ratio of the bending angle $\alpha_{0}$ to a real value of the bending angle $\alpha_{b}$ i.e., $k=\frac{\alpha_{0}}{\alpha_{b}}$. When the bent angle $\left(\alpha_{0}=k \alpha_{b}=\right.$ $\left.180^{\circ}\right)$, for example as in Franz (1961), Korzemski (1971), Śloderbach $(1999 ; 2002)$, then $k=\frac{180^{\circ}}{\alpha_{b}}$. If $\alpha_{0}=$ $90^{\circ}$, then $2 \alpha_{0}=k \alpha_{b}=180^{\circ}$, when $\alpha_{0}=60$, then $3 \alpha_{0}=k \alpha_{b}=180^{\circ}$, etc.

From the known tests of bending using the method of wrapping and the template and mandrel it appears that the coefficient $k$ decreases when the mandrel is well chosen, put forward and lubricated, and if the applied metals and their alloys are soft and very soft. In such a case, the angular range of the bending zone in the bent zone increases. On the contrary, from the tests of bending using the mandrel (Franz, 1961; Gruner, 1960; Grunow, 1985; Korzemski, 1968; 1971; Śloderbach, 1999; 2000; 2002) we can draw a conclusion that when the force of friction between the mandrel and the internal wall of the bent tube increases, and in the case of harder materials, the coefficient $k$ increases, too. In that case, the angular range of the bending zone decreases in the bent zone.

For the transformed and adapted for analytical calculations strains in external points of every $N$-th layer included in the bent tube wall in elongated and compressed layers the expressions Eq.(4.8) take the form

$$
\begin{aligned}
& \varphi_{1}^{(N)}=\lambda_{i} \ln \frac{2 R \pm d_{i}^{(N)} \cos \beta\left(\cos (k \alpha)-\cos \left(k \frac{\alpha_{b}}{2}\right)\right)}{2 R}, \\
& \varphi_{2}^{(N)}=\ln \frac{d_{i}^{(N)}}{d_{\mathrm{ext}}^{(N)}}, \quad \varphi_{3}^{(N)}=\ln \frac{g_{i}^{(N)}}{g_{w 0}^{(N)}}, \\
& g_{i}=\sum_{N=1}^{N=n} g_{l i}^{(N)} .
\end{aligned}
$$

and

From the assumptions and derived expressions Eqs (4.1)-(4.9) it appears that the bending angle $\alpha_{b}$ is the basic parameter determining the advancement of the bending process.

\subsection{Derivation of the expression for displacement of the neutral axis}

Ad.2. The aim is to derive an extended (for transient zones and for unfree bending) expression determining the displacement of the neutral axis of plastic bending. In paper (Tang, 2000) the author derived the following approximate expression for the displacement of the neutral axis

$$
y_{0}=\frac{0.42}{\tilde{r}} r_{m} .
$$

The extended expression determining the displacement of the neutral axis resulting from Chapter ad. 1 in this paper (with no derivation), valid for transient zones and not unbounded bending, is as follows 


$$
y_{0} \cong \lambda_{0} \frac{0.42}{\tilde{r}} r_{m}\left(\cos (k \alpha)-\cos \left(k \frac{\alpha_{b}}{2}\right)\right)
$$

where: $\lambda_{0}-$ correction coefficient of the displacement of the neutral layer, $\lambda_{0} \in\langle 0 ; 1\rangle, \tilde{r}$-relative radius of bending, $\tilde{r}=\frac{R}{d_{\text {ext }}}, r_{m}$-mean radius of the bent tube, $r_{m}=r_{\text {int }}+\frac{g_{0}}{2}$.

The coefficient $\lambda_{0}$ determines characteristic technological-material parameters of the tube bending process, such as: kind of mandrel, tube material, shape of the template and the flatter, strip pressure, clearances, forces of friction between the bent tube and the bending machine device, rigidity of the bending machine, kind of bending (cold, hot, self-hot, with preheating). From Eqs (4.10) and (4.11) it appears that for very small bending radii $R \in\left\langle 0.5 \times d_{\text {ext }} ; 1 \times d_{\text {ext }}\right\rangle$ and thinner-walled tubes $\left(s_{w}^{*}<<0.05\right)$, the maximum displacement of the neutral axis can be equal to $\sim 25 \%$ of a diameter value of the tube which is going to be bent. Greater displacements of the neutral axis can be caused by another bending technology because in the case of the considered ranges $\tilde{r}$ and $s_{w}^{*}$ tubes are often bent with the use of a force which is opposite to the force rotating the template so as to obtain a suitable stress distribution in the cross section. From the extended Eq.(4.11) it also appears that the displacement of the neutral axis is influenced not only by the bending radius and the tube thickness (thin-walled) (see Franz, 1961; Hill, 1986; Korzemski, 1968; 1971; Śloderbach, 1999; 2002; Wick et al., 2001; Yang and Lin, 2004), but by a suitable technology, bending parameters and the tube material, as well. From Eq.(4.11) it also appears that there are three additional parameters determining the displacement of the neutral axis and its position in the bending zone: the bending angle and the angle determining a position of the point in the bending zone, and the coefficient $k$. Thus, if $\left(\cos (k \alpha)=1\right.$ and $\left.\cos \left(k \frac{\alpha_{b}}{2}\right)=0\right)$, then $y_{0}=y_{\text {o } \max } \cong \lambda_{0} \frac{0,42}{\tilde{r}} r_{m}$, see Eq. (4.11).

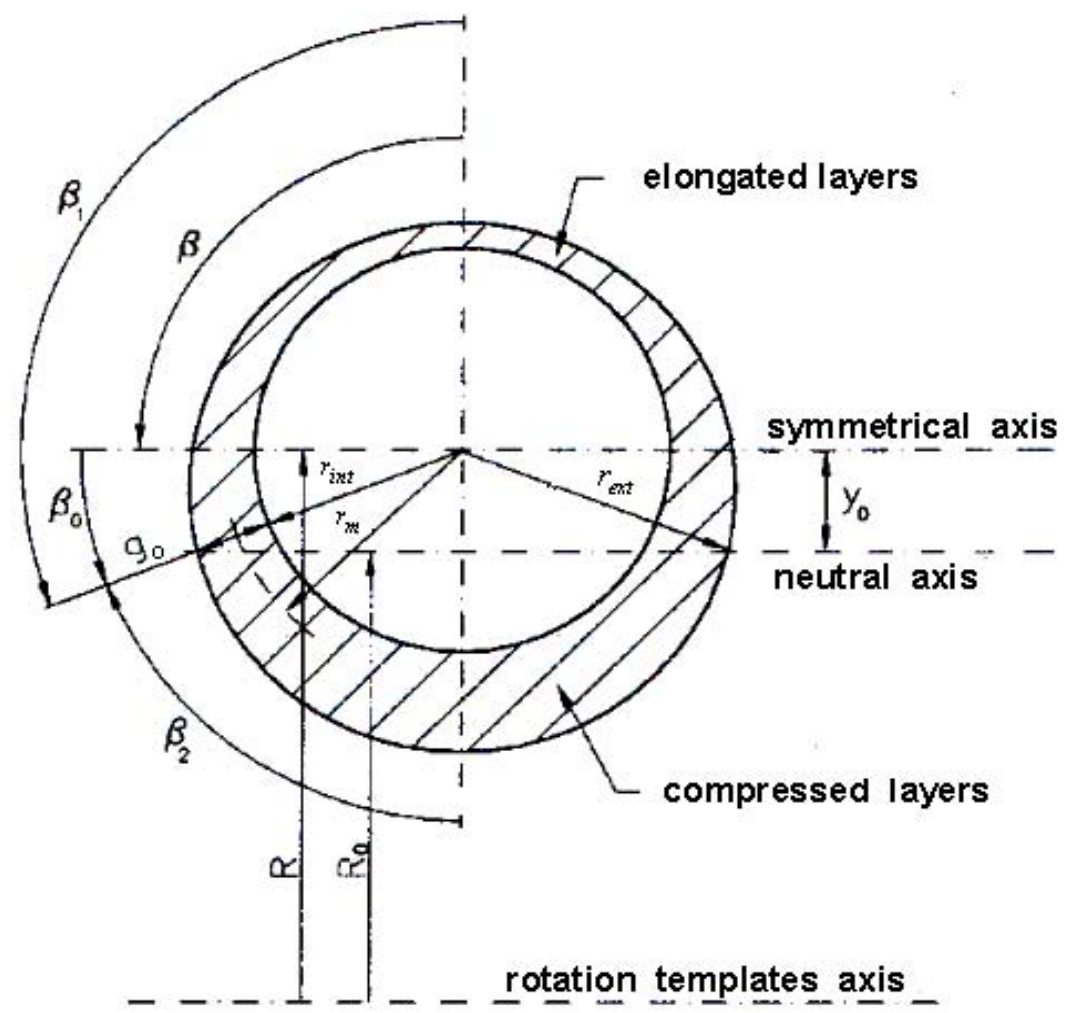

Fig.4. Schematic picture of the elbow cross-section and its characteristic parameters. 
Taking some additional calculations into account, in practice it is recommended to limit the considered bending method to the radii $\left(R \geq 1.5 \times d_{\text {ext }}\right)$. Pressure tubes which are most often used in pipelines for power industry and other tube installations of the devices of power engineering are usually in the range $\left(0.00<s_{w}^{*} \leq 0.125\right)$ or $\left(0.00<s^{*} \leq 0.100\right)$.

The introduced limitations concerning the tube bending parameters cause that, for example, the maximum (for instance for $R=1.0 \times d_{\mathrm{ext}}, s_{w}^{*}=0.03$ and $\lambda_{0}=0.5$ ) - relative (related to the external diameter of the bent tube) displacement of the neutral axis is $y_{0} / d_{\mathrm{ext}} \approx 10 \%$. However, for some ranges $\left(R\right.$ and $\left.s_{w}^{*}\right)$ and bending technologies and tube materials, relationships Eqs (4.8) or (4.9) which do not include the displacement of the neutral axis $y_{0}$ can be applied for strain description. Thus, they were applied for a precise description of fundamental experiments presented by (Franz, 1961). The estimated maximum value $y_{0}$, can be in practice even lower owing to a suitable selection and set-up of tooling of the bending machine, removal of clearances, a more plastic material for the bent tube, application of bending at elevated temperatures, increase of rigidity of the bending machine and so on. In the compressed layers, effects resulting from not unbounded upsetting may be less, they will be more intense along the perimeter of displacement of the bent tube material to the sides, upward and along the bent axis; it can cause lower values of the coefficient $\lambda_{0}$.

\subsection{Relationships including the displacement of the neutral axis}

Ad.3. The derived relationships for the strain state determination which describe the problem including the displacement of the neutral axis of plastic bending (according to Figs 2, 3 and 4) and according to items Ad.1 and Ad.2 by a suitable substitution of expression Eq.(4.11) determining $y_{0}$ for the modified Eqs (4.8) and (4.9) are as follows

\section{a). - for elongated lavers}

$$
\begin{aligned}
& \varphi_{1} \cong \lambda_{i} \ln \frac{2\left(R-y_{0}\right)+\left(d_{i} \cos \beta_{1}+2 y_{0}\right)\left(\cos (k \alpha)-\cos \left(k \frac{\alpha_{b}}{2}\right)\right)}{2\left(R-y_{0}\right)}, \\
& \varphi_{2} \cong \ln \frac{d_{i}}{d_{\text {ext }}}, \\
& \varphi_{3} \cong \ln \frac{g_{i}}{g_{0}}
\end{aligned}
$$

where: $\beta_{l^{-}}$directed angle of circulation of the elongated layers of the bent elbow, so as

$$
\beta_{1} \in\left\langle 0 ; 90^{\circ}+\beta_{0}\right\rangle,
$$

$\beta_{0}$ - angular range of the displacement of the neutral axis and $\sin \beta_{0}=\frac{y_{0}}{r_{\mathrm{ext}}} \approx \frac{y_{0}}{r_{m r}}$.

\section{b). - for compressed lavers}

$$
\begin{aligned}
& \varphi_{1} \cong \lambda_{i} \ln \frac{2\left(R-y_{0}\right)-\left(d_{i} \cos \beta_{2}-2 y_{0}\right)\left(\cos (k \alpha)-\cos \left(k \frac{\alpha_{b}}{2}\right)\right)}{2\left(R-y_{0}\right)}, \\
& \varphi_{2} \cong \ln \frac{d_{i}}{d_{\text {ext }}}, \\
& \varphi_{3} \cong \ln \frac{g_{i}}{g_{0}}
\end{aligned}
$$

where $\beta_{2}$ - directed angle of circulation of the compressed layers of the bent elbow, so as $\beta_{2} \in\left\langle 0 ; 90^{\circ}-\beta_{0}\right\rangle$. 
The systems of Eqs (4.12), (4.13) are the searched set of expressions for the description of the strain state in the bending process at bending machines (thin-walled metal tubes) with the method of wrapping at the rotating template and using the mandrel, or without a mandrel, but when $d_{i n} \approx$ const is kept while bending. The derived sets of equations include the effect of the displacement of the neutral axis of plastic bending on the deformation field. If in the sets Eqs (12) and (13) we substitute $\left(y_{0}=0\right)$, then the problem does not include the influence of the displacement of the neutral axis of plastic bending on the strain distribution. When, for example, $\left(R=1.0 \times d_{\mathrm{ext}}, s_{w}^{*}=0.03\right.$ and $\left.\lambda_{0}=0.5\right)$, then $\left(y_{0 \max } / d_{\mathrm{ext}} \approx 10 \%\right)$, and then the calculated increments of longitudinal and equivalent strains including the effect of the displacement of the axis are by $\sim 20 \%$ greater as compared with the values obtained for the case which does not include the axis displacement $y_{0}$. It means that for some certain values of the bending radius $\left(R<1.5 \times d_{\mathrm{ext}}\right)$ and for thin-walled tubes when $\left(s_{w}^{*} \leq 0.03\right)$, displacements of the neutral axis should not be neglected in the analytic description of the strain state. The estimated maximum value of $y_{0} / d_{\text {ext }}$ can be lower when, for example, $\left(R \geq 1.5 \times d_{\text {ext }}\right)$ and $\left(0.03 \leq s_{w}^{*}<0.125\right)$, then $\left(y_{0 \max } / d_{\mathrm{ext}} \approx 6 \%\right.$ and less). This is an additional reason why the relationship Eq.(2.8) can be used for the analysis and description of experimental results included in a fundamental paper (Franz, 1961), not including displacement of the neutral axis. Relationships Eq.(2.8) used in Śloderbach $(1999 ; 2000)$ do not include the displacement of the neutral axis $y_{0}$, because the bent tube tested in (Franz, 1961) was thick-walled where $s_{w}^{*} \approx 0.127$, then $\left(s^{*} \approx 0.1\right)$, and it was bent at the radius $R \cong 1.73 \times d_{\text {ext }}$ (so $R>$ $\left.1.5 \times d_{\mathrm{ext}}\right)$. Then for $\lambda_{0}=0.5, y_{0 \max } \approx 2.43 \mathrm{~mm}$ and $y_{0 \max } / d_{\mathrm{ext}} \approx 5.5 \%$.

Expressions (4.12) - (4.13) can be written in a more compact form as

$$
\begin{aligned}
& \varphi_{1} \cong \lambda_{i} \ln \frac{2\left(R-y_{0}\right) \pm\left(d_{i} \cos \beta_{1} \pm 2 y_{0}\right)\left(\cos (k \alpha)-\cos \left(k \frac{\alpha_{g}}{2}\right)\right)}{2\left(R-y_{0}\right)}, \\
& \varphi_{2} \cong \ln \frac{d_{i}}{d_{\text {ext }}}, \\
& \varphi_{3} \cong \ln \frac{g_{i}}{g_{0}} .
\end{aligned}
$$

According to the assumptions that the derived expressions for strain components in tube bending processes are identified with plastic strains (it appears that in the angular measure elastic strains are related to the main bending angle equal to some degrees (Franz, 1961; Korzemski 1971; Śloderbach, 1999; 2000; 2002), we obtain

$$
\varphi_{1}=\varphi_{1}^{p}, \quad \varphi_{2}=\varphi_{2}^{p}, \quad \varphi_{3}=\varphi_{3}^{p}, \quad \varphi_{(i)}=\varphi_{(i)}^{p},
$$

and

$$
\varepsilon_{l}=\varepsilon_{1}^{p}, \quad \varepsilon_{2}=\varepsilon_{2}^{p}, \quad \varepsilon_{3}=\varepsilon_{3}^{p}, \quad \varepsilon_{(i)}=\varepsilon_{(i)}^{p} .
$$

\section{Initial and boundary conditions for the generalized model of strains}

Expressions (4.12)- (4.14) satisfy the following boundary and initial conditions for the tube bending process

a) when $\alpha=\frac{\alpha_{b}}{2}=0$ - beginning of the bending process (no bending),

b) when $\alpha=\frac{\alpha_{b}}{2} \neq 0$ - beginning and end of the bending zone, 
c) when $\left(\beta_{1}=90^{\circ}+\beta_{0}\right.$ and $\left.\beta_{2}=90^{\circ}-\beta_{0}\right)$ - position of the layer of zero elongations (the neutral axis of plastic bending) defined by the radius $R_{0}$ in the bent zone. Then

$$
R_{i}=R_{0}, \quad r_{i}=r_{\mathrm{ext}} \quad \text { and } \quad g_{i}=g_{0},
$$

and

$$
\varphi_{1}=\varphi_{2}=\varphi_{3}=\varepsilon_{1}=\varepsilon_{2}=\varepsilon_{3}=0, \quad \text { and also } \quad \varphi_{(i)}=\varepsilon_{(i)}=0,
$$

d) when $(k \alpha)=\beta_{1}=\beta_{2}=0^{\circ}$ - tip points of the bending zone, and $\left(k \alpha_{b}\right) \in\left(0^{\circ} ; 180^{\circ}\right)$,

$$
\text { then }: R_{i}=\left(R-y_{0}\right) \pm\left(r_{\mathrm{int}}+g_{i} \pm y_{0}\right) \cdot\left(1-\cos \left(k \frac{\alpha_{b}}{2}\right)\right), \quad r_{i}=r_{\mathrm{int}}+g_{i}
$$

e) when: $(k \alpha)=\beta_{1}=\beta_{2}=0^{\circ}$ and $\left(k \alpha_{b}\right)=180^{\circ}$,

then $R_{i}$, and $\left(r_{i}, g_{i}\right)$ reach their extreme values, maximum and minimum (in elongated layers) or minimum and maximum (in compressed layers), respectively. It is a condition of initiation of the maximum strains at that point, and formation of the plateau zone, see (Franz, 1961; Korzemski 1971; Śloderbach, 1999; 2000; 2002)

\section{$\underline{\text { Zone of elongated layers }}$}

$$
\begin{aligned}
& g_{l}=g_{1 \text { min }}, \\
& R_{l}=R-y_{0}+\left(r_{\mathrm{int}}+g_{\min }+y_{0}\right), \\
& r_{l}=r_{\text {int }}+g_{l \text { min }} .
\end{aligned}
$$

\section{$\underline{\text { Zone of compressed layers }}$}

$$
\begin{aligned}
& g_{2}=g_{2 \max }, \\
& R_{2}=R-y_{0}-\left(r_{\text {int }}+g_{\text {max }}-y_{0}\right), \\
& r_{2}=r_{\text {int }}+g_{2 \max } .
\end{aligned}
$$

In this case, the main components of logarithmic and relative strains and their intensities also reach the extreme values which differ in the zones of elongated layers and compressed layers, respectively.

f) when $d_{i}=d_{\text {int }}=d_{\text {ext }}$ (internal surface of the bent tube), then - according to expressions Eqs (4.12) -(4.14)it appears that $\varphi_{2}=0 \Rightarrow \varepsilon_{2}=0$.

Note: The derived expressions $(12)_{1}-(14)_{1 \mathrm{i} 2}$, have a physical sense when the conditions for $R>y_{0}$ or for $R>$ $y_{0 \max }$, are satisfied, and in practice they are always satisfied.

\section{Exemplary calculations}

This chapter presents exemplary calculations of variation of longitudinal strains and wall thickness, including the displacement of the neutral axis of plastic bending related to the external diameter of the bent tube from the range from $0 \%$ to the maximum value $25 \%$. Simulation calculations were performed for a metallic (steel) tube of dimensions $\left(\phi 44.5 \times 4.5 \mathrm{~mm}\right.$ and $\left.s_{w}^{*} \approx 0.127\right)$ in the main bending plane in elongated layers $\left(\alpha=\beta_{1}=0^{\circ}\right)$, for the bending angle $\left(\alpha_{0}=k \alpha_{b}=180^{\circ}\right)$. For calculations, the following values of the technological-material coefficients were assumed: $k \cong 3$ and $\lambda_{1} \cong 1$ and the bending radius $R \approx 77 \mathrm{~mm}(R \approx$ $1.73 \times d_{\text {ext }}$ ). The data for calculations were taken from (Franz, 1961). The calculations were performed using expressions Eq.(4.14) and the condition of plastic incompressibility of the bent tube material. The calculation results are presented in Figs 5 and 6. 


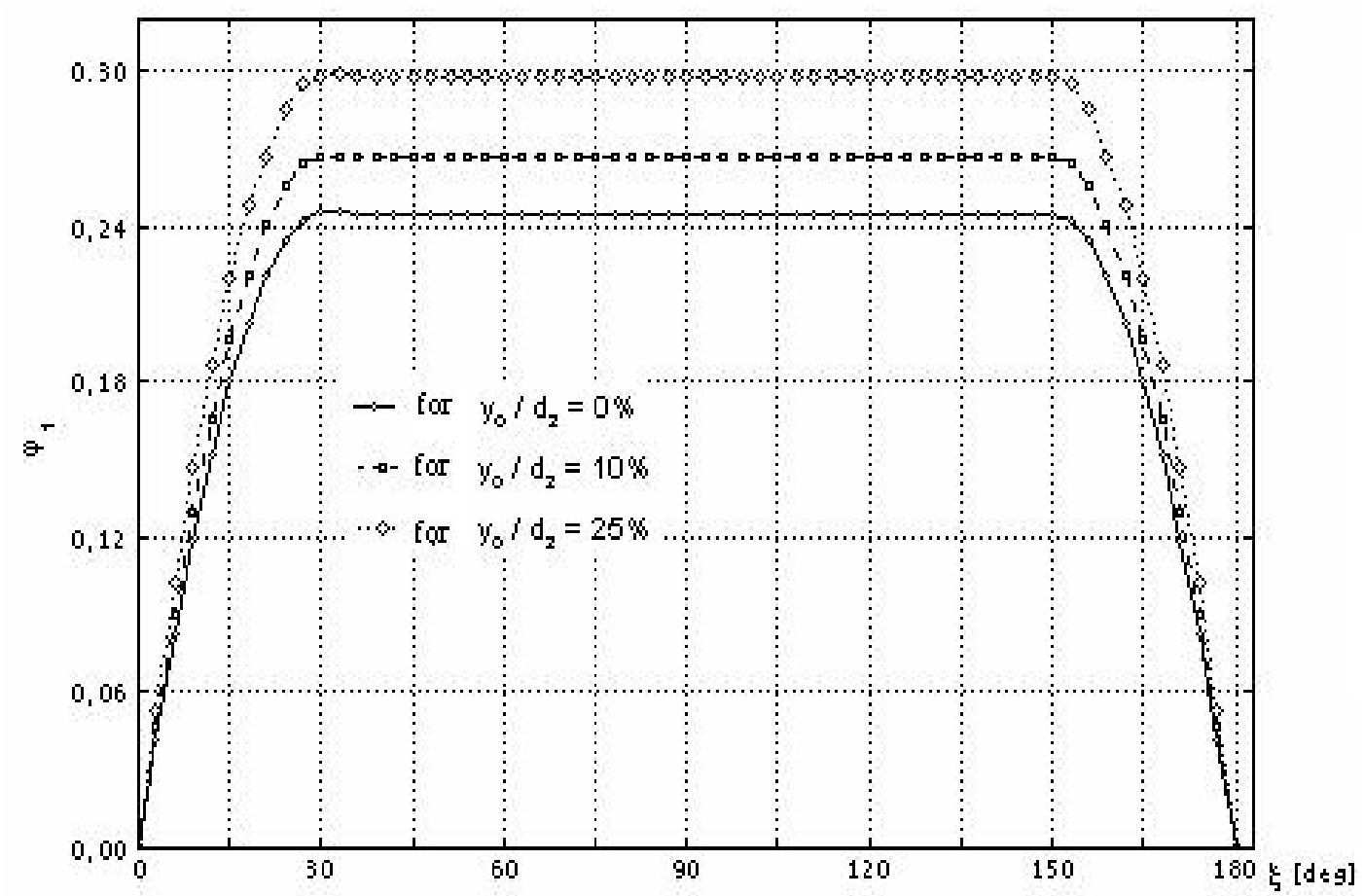

Fig.5. Exemplary calculations of longitudinal deformations, depending on the neutral axis displacement $y_{0}$, where $\left(d_{z} \equiv d_{\text {ext. }}\right)$.

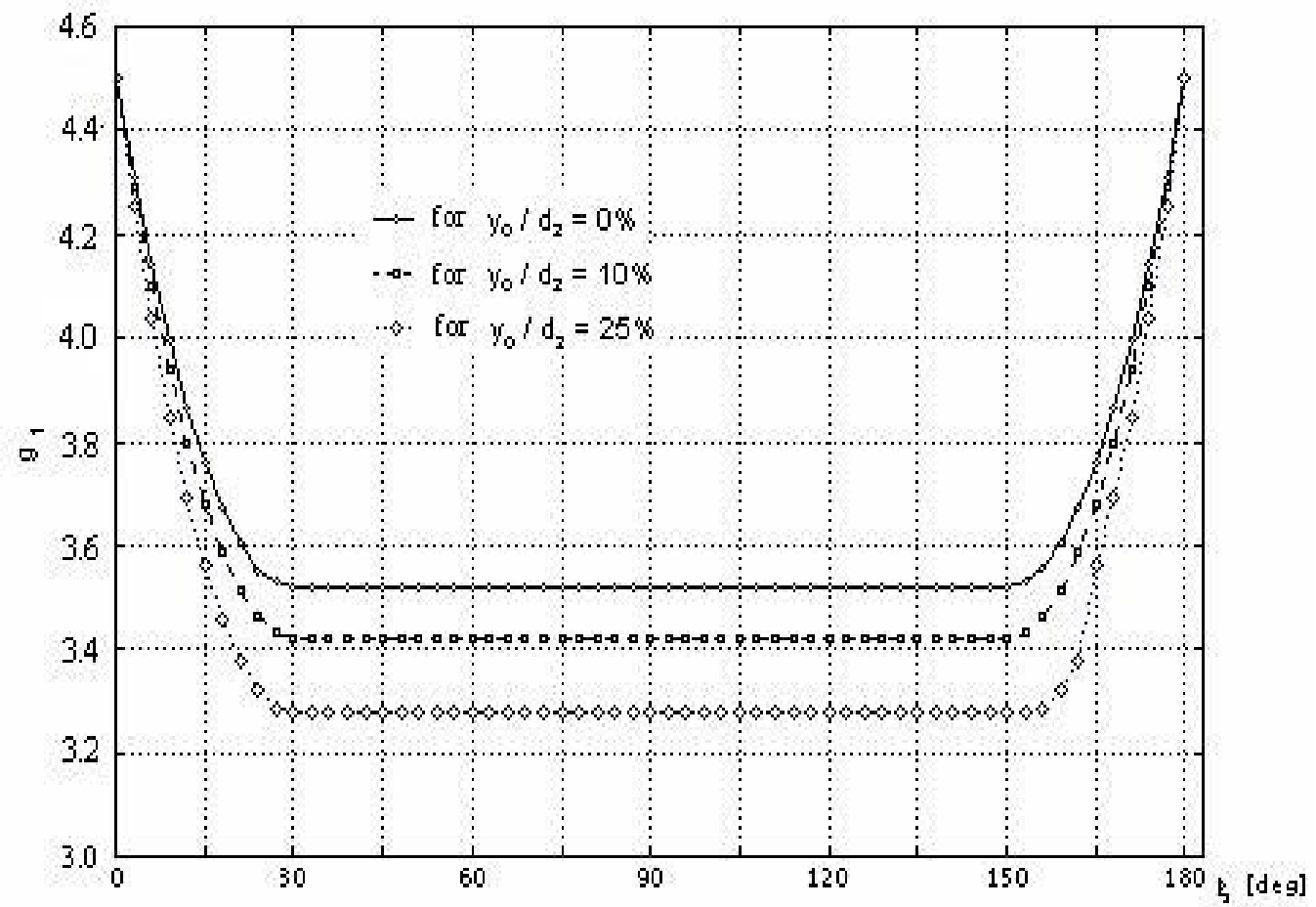

Fig.6. Exemplary calculations of the wall thickes distribution as depending on the neutral axis displacement $y_{0}$, where $\left(d_{z} \equiv d_{\text {ext. }}\right)$. 


\section{Conclusions}

1. The paper contains a derivation of the generalized relationships for logarithmic and relative measures of strains: longitudinal (along the tube axis), circumferential and along the thickness (radial) during bending thin- and thick-walled metallic tubes at bending machines. Generalization of strain description as compared to the previous papers by the author (Śloderbach, 1999; Śloderbach and Rechul, 2000) consists in including the displacement of the neutral axis. The strains can be defined in the main bending plane and each parallel or perpendicular plane, i.e., in all points of the bending zone. The derived relationships describing the measures of logarithmic and relative strains depend on the bending radius $R$, geometrical dimensions of the tube, the bending angle $\alpha_{b}$, angular coordinates $\alpha$ and $\beta$, which describe the bending zone in the range of the bending angle $k \alpha_{b} \in<0^{\circ} ; 180^{\circ}>$, displacement of the neutral axis $y_{0}$ and two technological-material coefficients $k$ and $\lambda_{i}$. The results of exemplary calculations of longitudinal strains and wall thickness distribution for the elongated layers performed for the bending angle $\left(\alpha_{0}=k \alpha_{b}=180^{\circ}\right)$ including the displacement of the neutral axis related to the external diameter from the range from $0 \%$ to the maximum value $25 \%$, were shown in the form of curves. The calculations included a value of the coefficient of the bending zone range $(k=3)$ and the coefficient of strain distribution in the elongated layers $\left(\lambda_{1}=1\right)$. From the graphs it appears that there is a certain proportionality between the values of the displacement of the neutral axis expressed in $(\%)$, and relative increment of the longitudinal strain and relative reduction of the elbow wall thickness.

2. In future tests an explicit (analytic) form of $k$ depending on the coefficient of friction and suitable technological- material parameters of bending can be searched. When the coefficient of friction between the mandrel and the internal wall of the bent tube tends to infinity, the coefficient $k$ tends to infinity, too. It means no bending because the angular range of the bending zone tends to zero $\left(\alpha_{b} \rightarrow 0^{\circ}\right)$. Then, also the angle of bend zone tends to zero $\left(\alpha_{0} \rightarrow 0^{\circ}\right)$.

3. While considering on the problems including the displacement of the neutral axis of plastic bending some simplifications can be introduced, for example neglecting $y_{0}$ in the first or second term in the numerator in expressions (4.12) $)_{1}$ and (4.13) $)_{1}$ or the numerators or denominators of expressions $(4.12)_{1}$ and $(4.13)_{1}$ in order to obtain the best conformity of the calculated quantities with the experimental data or those taken from literature. Such simplifications should be dependent on bending parameters occurring in Eqs (4.10) and (4.11).

4. The paper (Śloderbach, 1999) presents the results of the analytical and numerical calculations based on the derived relationships Eqs (4.12)-(4.14). The results of calculations coincide with the experimental data from (Franz, 1961) for the tube of dimensions $\left(\phi 44.5 \times 4.5 \mathrm{~mm}\right.$ and $\left.s_{w}^{*} \approx 0.127\right)$, the bending radius $R$ $\approx 77 \mathrm{~mm}\left(R \approx 1.73 \times d_{\mathrm{ext}}\right)$. The calculation results have been shown in the form of appropriate graphs.

\section{Nomenclature}

$$
\begin{aligned}
& d_{i}-\text { local actual external diameter of the elbow in the bending zone } \\
& d_{\text {ext }} \text { and } d_{\text {int }}-\text { external and internal diameter of a bent tube } \\
& d_{\text {lint }}^{(k)}, d_{\text {ext }}^{(k)} \quad \text { - internal and external diameter of the } k \text {-th layer, respectively } \\
& d_{\text {ext }} \text { and } d_{\text {int }}-\text { external and internal diameter of the tube for bending, respectively } \\
& g_{0} \text { - initial thickness of a bent tube } \\
& g_{i}-\text { actual thickness of a bend within the bending zone ( } i=1 \text { for elongated layers, } i=2 \text { for compressed } \\
& g_{0}^{(k)} \quad \text { - thickness of the } k \text {-th layer measured from } d_{\text {int }} \\
& g_{l 0}^{(I)}, g_{l 0}^{(k)}, g_{i 0}^{(n)} \quad \text { - thickness of the } 1 \text {-st, } k \text {-th and } n \text {-th (last) layer } \\
& k \text { - technological-material coefficient dependent on the bent tube material and the applied bending }
\end{aligned}
$$




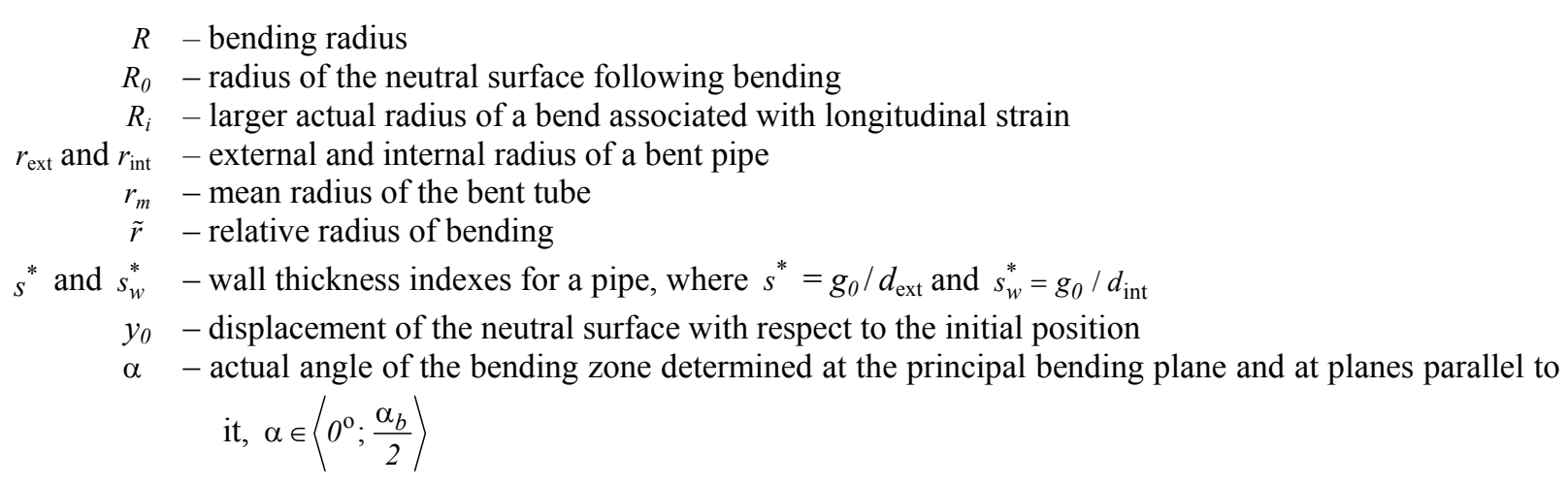

where $\alpha_{b}$ - bending angle measured over the bending zone, $\alpha_{b} \in\left\langle 0^{\circ} ; 180^{\circ}\right\rangle$,

$\alpha_{0}$ - bend angle (the angle by which a template or a farmer is rotated); in theory for spirals $\alpha_{0} \in\left\langle 0^{\circ} ; \infty\right.$ ) but for the method under analysis $\alpha_{0} \in\left\langle 0^{\circ}, 180^{\circ}\right\rangle$. Obviously, within the bending zone the two angles are equal $\left(\alpha_{0}=\alpha_{b}\right)$. When the plateau zone was formed, then $\left(\alpha_{0}=\alpha_{b}+\alpha_{p l}\right)$, where $\alpha_{p l}$-angle of a plateau zone (Śloderbach, 2002).

$\beta$ - actual angle determined at the planes perpendicular to the bending plane, that $\beta \in\left\langle 0^{\circ}, 90^{\circ}\right\rangle$

$\beta_{2}$ - directed angle of circulation of the compressed layers of the bent elbow

$\beta_{0}$ - angular range of the displacement of the neutral axis

$\varepsilon_{1}, \varepsilon_{2}, \varepsilon_{3}$ - relative components of plastic deformations

$\varphi_{1}, \varphi_{2}, \varphi_{3}$ - logarithmic (true) components of plastic deformations

$\lambda_{i}$ - technological-material coefficient of strain distribution in the elongated layers $(i=1)$ and compressed layers $(i=2)$

$\lambda_{0}-$ correction coefficient of the displacement of the neutral layer, $\lambda_{0} \in\langle 0 ; 1\rangle$

\section{References}

Beskin L. (1945): Bending of thin curved tubes. - Journal of Applied Mechanics, Transactions of the ASME, vol.12.

Boyle M. (1971): Bending thin wall stainless tubing. - Machinery, No77/71, London.

European Standard EN 448. (1993): Manufacturing.

Franz W.D. (1961): Das Kalt-Biegen von Rohren. - Berlin: Springer-Verlag.

Franz W.D. (1969): Numerisch gesteuerte Rohrkaltbiegemaschinen. - Werkstatt und Betrieb, Heft vol.9/69, pp.129145.

Gruner P. (1960): Über Rohrbiegeverfahren. - Maschinenmarkt, vol.30/31, pp.120-129.

Grunow O. (1985): Praktisches Rohrbiegen. - Berlin: Springer-Verlag.

Hill R. (1986): Mathematical Theory of Plasticity. - London-Oxford: Clarendon Press.

Johnson W., Mellor P.B. (1975): Engineering Plasticity. - London: van Nostrand Reinhold Company.

Korzemski J.W. (1968): Thin-walled pipe bending with use of mandrels [in Polish]. - Mechanic, vol.4/68, Warsaw, pp.207-210.

Korzemski J.W. (1971): Thin-Walled Pipes Bending [in Polish]. - Warsaw: WNT.

Li H., Yang H., Zhan M. and Gu R.J. (2006): A new method to accurately obtain wrinkling limit diagram in NC bending process of thin-walled tube with large diameter under different loading paths. - Journal of Materials Processing Technology, vol.177, pp.192-196.

Marciniak Z. (1971): Limit Deformations in Sheet Metal Stamping [in Polish]. - Warsaw: WNT.

Mendelson A., (1988): Plasticity-Theory and Applications. - New York: Mc Millan Company.

Olszak W., Perzyna P. and Sawczuk A. (1985): Theory of Plasticity [in Polish]: Warsaw: PWN. 
Pesak F. (1953): Bending thin wall tubing. - Machinery, vol.60, pp.147-151.

Szczepinski W. (1973): Theory of Plastic Working of Metals [in Polish]. - Warsaw: PWN.

Śloderbach Z. (1999): A model for strain geometry evaluation in pipe bending processes. - Engineering Transactions, vol.47,1, pp.3-20.

Śloderbach Z. (2002): Some problems of mechanics in pipeline bending processes [in Polish]. - Publishing House of Wrocław University of Technology, ISBN 83-7085-665-9, Wrocław, pp.1-218.

Śloderbach Z. (2012): Application of the new flexible multi-segment mandrel for bending metallic tubes on machine benders [in Polish]. - Power Industrty, (Statement Notebook), No XXIV, pp.97-99.

Śloderbach Z. and Rechul Z. (2000): Effect of strain hardening and normal anisotropy on admissible values of strain and stress in pipe-bending processes. - Journal of Theoretical and Applied Mechanics, vol.4, 38, pp.843-859.

Śloderbach Z. and Rechul Z. (2006): A thermodynamic approach to the stored energy concept. - Journal of Technical Physics, vol.XLVII, pp.83-102.

Śloderbach Z., Pająk J. and Marciniak Z. (2008): Apriximate calculations of the stored energy of plastic deformation in tube bending processes. - Optmization of Production Processes, Studies and Monographs, No.238, Opole University of Technology, pp.169-185.

Tang N.C. (2000): Plastic-deformation analysis in tube bending. - International Journal of Pressure Vessels and Piping, vol.77, No.12, Publisher: Elsevier, UK, pp.751-759.

UDT Conditions (WUDT-UC-WO-O/02:10). (2003): Pressure Installations. General Requirements. Strength Calculations [in Polish]. - Issue I, Warsaw.

Wick C., Benedict J.T. and Veilleux R.F. (2001): Tool and manufacturing engineers handbook. A reference book for manufacturing engineers, managers and technicans, Volume II, Forming, Fourth Edition. - Society of Manufacturing Engineers, One SME Drive, Dearbon, Michigan, USA.

Yang H. and Lin Y. (2004): Wrinkling analysis for forming limit of tube bending processes. - Journal of Materials Processing Technology, vol.152, pp.363-369.

Zdankiewicz M. (1970): Investigations of the cold tubes bending processes [in Polish]. - Mechanic, No 7/70, Warsaw, pp.609-519.

Zdankiewicz M. (1998): European Direktive concern a pressure installations. Requirements concern a manufacturing [in Polish]. - Technical Inspection, vol.2/ 98, Warsaw, pp.25-33 and 48.

Zhang Z., Yang H., Li H., Ren N. and Tian Y. (2011): Bending behaviors of large diameter thinwalled CP-Ti tube in rotary draw bending. - Progress in Natural Science: Materials International, vol.21, pp.401-412.

Zhiqiang J., Mei Z., He Y., Xudong X. and Guangjun L. (2011): Deformation behavior of mediumstrength TA18 highpressure tubes during $n c$ bending with different bending radii. - Chinese Journal of Aeronautics, vol.24, pp.657664.

Życzkowski M. (1981): Complex loading in theory of plasticity. - PWN, Nijhoff, Warsaw, Alphen aan den Rijn, (extended edition).

Received: September 5, 2013

Revised: October 20, 2013 